\section{Importancia del proceso de limpieza y desinfección de superficies críticas en un servicio dental. Impacto de un programa de intervención}

Elena Véliz, Teresa Vergara, Mercedes Pearcy y Jeannette Dabanch

\author{
Importance of cleaning and disinfection of critical \\ surfaces in dental health services. Impact of an \\ intervention program
}

Introduction: Dental care has become a challenge for healthcare associated infection prevention programs, since the environment, within other factors, plays an important role in the transmission chain. Materials and Methods: An intervention program was designed for the Dental Unit of Hospital Militar de Santiago, between years 2014 and 2015. The program contemplated 3 stages: diagnostic, intervention and evaluation stage. Objective: To improve the safety of critical surfaces involved in dental healthcare. Results: During the diagnostic stage, the cleaning and disinfection process was found to be deficient. The most contaminated critical surface was the instrument holder unit, then the clean area and lamp handle. The surfaces that significantly reduced their contamination, after the intervention, were the clean area and the instrument carrier unit. Conclusion: Training in the processes of cleaning and disinfecting surfaces and dental equipment is one of the cost-effective strategies in preventing healthcare-associated infections (HCAI), with simple and easy-to-apply methods.

Key words: Cleaning and disinfection of surfaces; infections in dental care; bioluminescence.

Palabras clave: Limpieza y desinfección de superficies; infecciones en atención dental; bioluminiscencia.

\section{Introducción}

$\mathrm{L}$ a atención odontológica se ha transformado en un desafío para los programas de prevención y control de infecciones asociadas a la atención de salud (IAAS). En esta práctica se ha documentado la transmisión de diversos agentes infecciosos entre pacientes y personal de salud durante la atención'.

El ambiente, entre otros factores, juega un rol importante en la cadena de transmisión, dado que los agentes microbiológicos tienen la capacidad de sobrevivir por períodos prolongados en las superficies inanimadas. Por otro lado, la cavidad oral actúa también como un nicho ecológico para microorganismos resistentes a los antimicrobianos ${ }^{2}$.

Unidad de Infecciones Asociadas a la Atención de Salud, Hospital Militar de Santiago.

Conflicto de interés: ninguno

Financiamiento: ninguno

Recibido: 9 de mayo de 2017 / Aceptado: 23 de noviembre de 2017

Correspondencia a:

Elena Véliz G.

eveliz@hms.cl
Durante los procedimientos dentales se produce el contacto directo entre la mucosa oral y las manos del odontólogo, contacto con fluidos del paciente y generación de aerosoles en los procedimientos, ocasionando la contaminación de las distintas superficies y equipos que serán utilizados posteriormente con otros pacientes.

En el Servicio de Dental de nuestro hospital se pesquisaron deficiencias en la limpieza y desinfección de las superficies de trabajo, a través de la aplicación del método de bioluminiscencia, lo cual motivó realizar un programa de intervención.

\section{Material y Método}

Se realizó un programa de intervención en el Servicio de Dental del Hospital Militar de Santiago, entre los años 2014 y 2015, cuyo objetivo fue mejorar las condiciones de seguridad de las superficies críticas involucradas en la atención dental a través de la limpieza y desinfección de superficies. Este programa contempló tres etapas:

\section{Etapa diagnóstica}

Medición de la limpieza de las superficies críticas por medio de la cuantificación de adenosin trifosfato (ATP) por el método de bioluminiscencia. Las superficies críticas se definieron en base a la frecuencia de manipulación de éstas (más de cuatro veces durante la atención) ${ }^{3}$. Según este criterio se seleccionó la manilla de la lámpara de luz intra-oral, la unidad porta-instrumental y el área limpia (Figura 1).

Para la medición de ATP se tomó como punto de corte para una superficie limpia y segura una lectura de 250 URL y menos ${ }^{4}$.

\section{Etapa de intervención}

Contempló las siguientes estrategias:

- Protocolo de limpieza con solución jabonosa y desinfección con alcohol $70 \%$ de las superficies posterior a la atención de cada paciente de acuerdo a la evidencia ${ }^{5}$ y de los ductos internos de las unidades dentales al finalizar cada jornada, con una solución recomendada por el fabricante.

- Capacitación al equipo de enfermería con un taller de demostración de limpieza y desinfección de las superficies a cargo de la Unidad de Prevención y Control de IAAS.

- Capacitación al equipo de enfermería en limpieza de ductos internos de unidad dental a cargo de una empresa externa. Este proceso se realizó al final de cada jornada.

\section{Etapa de evaluación}

Medición de las superficies críticas después de la limpieza por cuantificación de ATP, al igual que en la etapa diagnóstica. La técnica consistió en tomar la tórula por la parte plástica superior y sin tocar la parte blanca, hacer rodar el hisopo en forma horizontal de un lado hacia el otro por sobre la superficie y luego verticalmente. Posteriormente se introdujo la tórula al tubo presionando la tapa, moviendo suavemente y se colocó dentro del bioluminómetro para la medición de la muestra.

\section{Resultados}

Durante el programa de intervención se hizo medición de ATP en todas las superficies de los cubículos de atención. En total se tomaron 131 muestras, 65 en la etapa diagnóstica y 66 en la de evaluación. 


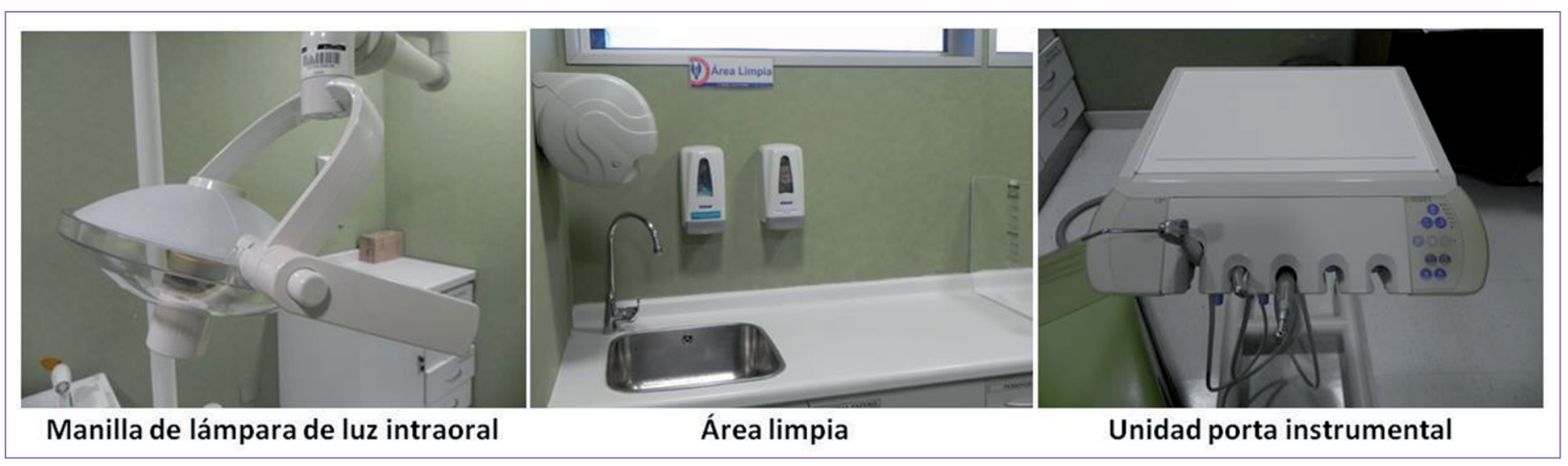

Figura 1. Superficies críticas en la atención dental.

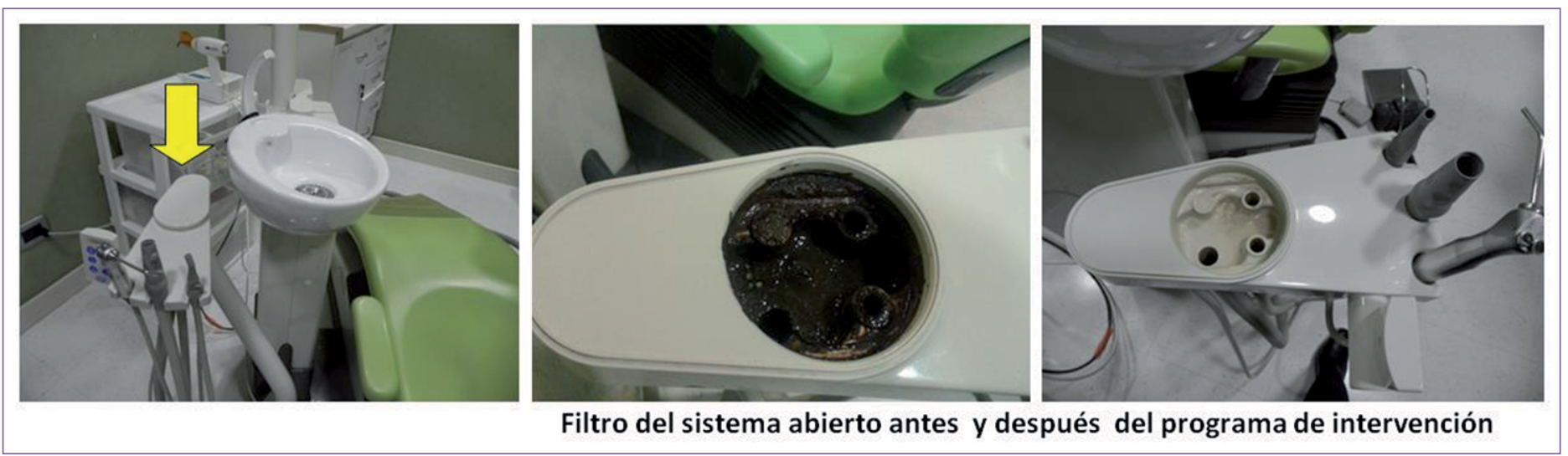

Figura 2. Filtro del sistema rotatorio y jeringa triple función.

En la etapa diagnóstica se pudieron constatar deficiencias en el proceso de limpieza y desinfección, donde la superficie crítica más contaminada fue la unidad porta-instrumental (28\% bajo 250 URL), luego el área limpia (38\% bajo $250 \mathrm{URL})$ y posteriormente la manilla de la lámpara $(52 \%$ bajo 250 URL). Como parte de la intervención se capacitó a todo el equipo de enfermería, el cual fue evaluado con una prueba escrita sobre la técnica del proceso de limpieza y desinfección. Las superficies que mejoraron con posterioridad a la intervención fueron el área limpia (38\% vs 86\% de cumplimiento, $\mathrm{p}=0,005)$ y la unidad porta-instrumental $(28 \%$ vs $68 \%$ de cumplimiento $\mathrm{p}=0,023$ ) con una diferencia significativa en la reducción de la contaminación. La manilla de la lámpara también mejoró sus condiciones de limpieza; sin embargo, la diferencia no fue estadísticamente significativa ( $52 \%$ vs $81 \% \mathrm{p}=0,07$ por prueba de Mann Whitney).

Adicionalmente se detectó, a la inspección visual, sarro y suciedad visible en los ductos internos de la unidad dental. La falta de limpieza de los ductos constituye un riesgo de transmisión de microorganismos dado que estos se contaminan por el paso de agua aspirada desde la cavidad oral.

No se realizaron cultivos microbiológicos de los ductos internos en ninguna etapa del programa, sin embargo, la evaluación visual permitió evidenciar mejoría posterior a la intervención (Figura 2).

\section{Discusión}

La capacitación en relación a los procesos de limpieza y desinfección de superficies y del equipamiento dental es una de las estrategias costoeficientes en la prevención de IAAS, sencilla y fácil de aplicar ${ }^{6}$.

El monitoreo a través de la medición de ATP es un sistema sencillo y rápido ${ }^{7}$ que permite validar de manera objetiva el proceso de limpieza, refuerza los logros del equipo y permite concientizar al personal en la importancia de este proceso.

La limpieza y desinfección es esencial para la prevención de $\operatorname{IAAS}^{8}$ y debe ser focalizado en las zonas de mayor contaminación. La literatura especializada disponible sólo define estas áreas en las unidades críticas ${ }^{9}$. Por ello, nosotros consideramos la manilla de la lámpara de luz intra-oral, la unidad porta-instrumental y el área limpia como áreas críticas en la atención dental.

Es de gran relevancia contar con un programa de limpieza y desinfección de los ductos internos de las unidades dentales, donde lo óptimo es cuantificar las unidades formadoras de colonias (ufc) y que éstas se encuentren bajo el rango establecido ${ }^{10}$.

En la presente investigación pudimos documentar el impacto del proceso de limpieza y desinfección de superficies a través de la capacitación 
y monitorización, lo cual sin duda fomenta un trabajo seguro. Estamos convencidos de que nuestra experiencia puede contribuir y motivar a otros equipos a mejorar la calidad de atención en esta área, dado la poca evidencia disponible.

Agradecimientos: Agradecemos el apoyo y compromiso de Jessica Parada, enfermera, Jerko Raffo, odontólogo jefe del Servicio MáxiloFacial y de todo el equipo de técnicos dentales que se desempeñan en esta área.

\section{Resumen}

Introducción: La atención odontológica se ha transformado en un desafío para los programas de prevención y control de infecciones asociadas a la atención de salud (IAAS), dado que el ambiente, entre otros factores, juega un rol importante en la cadena de transmisión. Método: Se realizó un programa de intervención en el Servicio de Dental del Hospital Militar de Santiago, entre los años 2014-2015, que contempló tres etapas: etapa diagnóstica, de intervención y de evaluación. Objetivo: Mejorar la seguridad de las superficies críticas involucradas en la atención dental. Resultados: Durante la etapa diagnóstica se constató que el proceso de limpieza y desinfección era deficiente. La superficie crítica más contaminada fue la unidad porta-instrumental, luego el área limpia y la manilla de la lámpara. Las superficies que redujeron significativamente su contaminación, posterior a la intervención, fueron el área limpia y la unidad porta-instrumental. Conclusiones: La capacitación en relación a los procesos de limpieza y desinfección de superficies y del equipamiento dental es una de las estrategias costo-eficientes en la prevención de las IAAS, sencilla y fácil de aplicar.

\section{Referencias bibliográficas}

1.- Ministerio de salud, Departamento de Calidad y Formación, Programa control de IAAS. Informe de Vigilancia de Infecciones Asociadas a la Atención en Salud, 2014. Disponible en: http://web.minsal.cl/wp-content/ uploads/2016/10/informe-IAAS-2014.pdf

2.- Kramer A, Schwebke I, Kampf G. How long do nosocomial pathogens persist on inanimate surfaces? A systematic review. BMC Infect Dis 2006; 6: 130.

3.- Weber D J, Rutala W A, Miller M B, Huslage K, Sickbert-Bennett E. Role of hospital surfaces in the transmission of emerging health care-associated pathogens: norovirus, Clostridium difficile, and Acinetobacter species. Am J Infect Control 2010; 38 (5 Suppl 1): S25-33.

4.- Lewis T, Griffith C, Gallo M, Weinbren M. A modified ATP benchmark for evaluating the cleaning of some hospital environmental surfaces. J Hosp Infect 2008; 69: 156-63.

5.- Centers for Disease Control and Prevention. Rutala W, Weber D and the Healthcare Infection Control Practices Advisory Committee (HICPAC). Guideline for Disinfection and Sterilization in Healthcare Facilities, 2008. Disponible en: https://www.cdc.gov/infectioncontrol/pdf/guidelines/ disinfection-guidelines.pdf

6.- Sjöberg M, Eriksson M. Hand disinfectant practice: the impact of an education intervention. Open Nurs J 2010; 4: 20-4.

7.- $\quad$ Sherlock O, O’Connell N, Creamer E, Humphreys H. Is it really clean? An evaluation of the efficacy of four methods for determining hospital cleanliness. J Hosp Infect 2009; 72: 140-6.

8.- Dancer S J. The role of environmental cleaning in the control of hospitalacquired infection. J Hosp Infect 2009; 73: 378-85.

9.- Ferreira M, Andrade D, Rigotti M, Ferrareze M. Condition of cleanliness of surfaces close to patients in an intensive care unit. Rev Latino-Am. Enfermagem 2011; 19: 557-64.

10.- Arraigada A, Larrucea C, Padilla C. Control de infección en los ductos de equipos dentales de las clínicas odontológicas de la Universidad de Talca. Rev Dent Chile 2004, 95:3-9. 